\title{
The 2005 Outburst of the Halo Black Hole X\#Ray Transient XTE J1118+480
}

\section{Citation}

Zurita, C., M. A. P. Torres, D. Steeghs, P. Rodriguez\#Gil, T. Munoz\#Darias, J. Casares, T. Shahbaz, et al. 2006. "The 2005 Outburst of the Halo Black Hole X\#Ray Transient XTE J1118+480." The Astrophysical Journal 644 (1): 432-38. https://doi.org/10.1086/503286.

\section{Permanent link}

http://nrs.harvard.edu/urn-3:HUL.InstRepos:41399946

\section{Terms of Use}

This article was downloaded from Harvard University's DASH repository, and is made available under the terms and conditions applicable to Other Posted Material, as set forth at http:// nrs.harvard.edu/urn-3:HUL.InstRepos:dash.current.terms-of-use\#LAA

\section{Share Your Story}

The Harvard community has made this article openly available.

Please share how this access benefits you. Submit a story.

Accessibility 
The Astrophysical Journal, 644:432-438, 2006 June 10

(C) 2006. The American Astronomical Society. All rights reserved. Printed in U.S.A.

\title{
THE 2005 OUTBURST OF THE HALO BLACK HOLE X-RAY TRANSIENT XTE J1118+480
}

\author{
C. ZuRITA \\ Instituto de Astrofísica de Canarias, 38200 La Laguna, Tenerife, Spain; czurita@iac.es \\ M. A. P. Torres and D. Steeghs \\ Harvard-Smithsonian Center for Astrophysics, 60 Garden Street, Cambridge, MA 02138 \\ P. Rodríguez-Gil, T. Muñoz-Darias, J. Casares, and T. Shahbaz \\ Instituto de Astrofísica de Canarias, 38200 La Laguna, Tenerife, Spain \\ I. G. MartíneZ-PAis \\ Instituto de Astrofísica de Canarias, 38200 La Laguna, Tenerife, Spain; and Departamento de Astrofísica, \\ Universidad de La Laguna, E-38206 La Laguna, Tenerife, Spain \\ P. ZHAO AND M. R. Garcia \\ Harvard-Smithsonian Center for Astrophysics, 60 Garden Street, Cambridge, MA 02138 \\ A. Piccioni, C. Bartolini, and A. Guarnieri \\ Dipartimento di Astronomia, Universitá di Bologna, Via Ranzani 1, Bologna, Italy \\ J. S. BLOOM \\ Astronomy Department, University of California, Berkeley, CA 94720 \\ C. H. Blake, E. E. Falco, and A. Szentgyorgyi \\ Harvard-Smithsonian Center for Astrophysics, 60 Garden Street, Cambridge, MA 02138 \\ AND \\ M. SKRUTSKIE \\ University of Virginia, Department of Astronomy, P.O. Box 3818, Charlottesville, VA 22903 \\ Received 2005 October 7; accepted 2006 February 8
}

\begin{abstract}
We present optical and infrared monitoring of the 2005 outburst of the halo black hole X-ray transient XTE $\mathrm{J} 1118+480$. We measured a total outburst amplitude of $\sim 5.7 \pm 0.1 \mathrm{mag}$ in the $R$ band and $\sim 5 \mathrm{mag}$ in the infrared $J$, $H$, and $K_{s}$ bands. The hardness ratio HR2 (5-12 keV : $\left.3-5 \mathrm{keV}\right)$ from the $R X T E$ ASM data is $1.53 \pm 0.02$ at the peak of the outburst, indicating a hard spectrum. Both the shape of the light curve and the ratio $L_{\mathrm{X}}(1-10 \mathrm{keV}) / L_{\mathrm{opt}}$ resemble the minioutbursts observed in GRO J0422+32 and XTE J1859+226. During early decline, we find a $0.02 \mathrm{mag}$ amplitude variation consistent with a superhump modulation, like the one observed during the 2000 outburst. Similarly, XTE J1118+480 displayed a double-humped ellipsoidal modulation distorted by a superhump wave when settled into a near-quiescence level, suggesting that the disk expanded to the 3:1 resonance radius after outburst, where it remained until early quiescence. The system reached quiescence at $R=19.02 \pm 0.03$, about 3 months after the onset of the outburst. The optical rise preceded the X-ray rise by at most 4 days. The spectral energy distributions (SEDs) at the different epochs during outburst are all quasi-power laws with $F_{\nu} \propto \nu^{\alpha}$ increasing toward the blue. At the peak of the outburst, we derived $\alpha=0.49 \pm 0.04$ for the optical data alone and $\alpha=0.1 \pm 0.1$ when fitting solely the infrared. This difference between the optical and the infrared SEDs suggests that the infrared is dominated by a different component (a jet?), whereas the optical is presumably showing the disk evolution.
\end{abstract}

Subject headings: accretion, accretion disks - binaries: close -

stars: individual (XTE J1118+480, KV UMa) - X-rays: stars

\section{INTRODUCTION}

X-ray transients (XRTs) are a class of low-mass X-ray binaries in which long periods of quiescence (typically decades) are interrupted by dramatic outbursts, when the X-ray luminosity suddenly increases by up to a factor of $10^{6}$ (e.g., Charles \& Coe 2006). During the outburst, XRTs usually reach a state in which the X-ray emission is dominated by thermal emission from the hot inner accretion disk (i.e., the high/soft [HS] or thermaldominated state). There are, however, a few of these systems (see, e.g., Brocksopp et al. 2004) that are instead dominated by a hard nonthermal power-law component, likely produced by thermal Comptonization of seed photons in the vicinity of the accreting black hole (i.e., the low/hard [LH] state). At even lower accretion rates, XRTs reach quiescence, which may be just an extreme example of the LH state. To explain the LH (and quiescent) state, a standard disk truncated at some large inner radius is assumed. The interior volume is filled with a hot, optically thin, quasi-spherical accretion flow, where most of the energy released via viscous dissipation remains in this flow rather than being radiated away (as in a disk) to be finally advected by the compact object (e.g., Narayan et al. 1996). This model, called advection-dominated accretion flow (ADAF), is the most widely discussed picture, although other alternatives have also been invoked. For instance, the accretion disk corona model assumes a cool thin disk embedded in a hot corona powered by magnetic flares (e.g., Merloni \& Fabian 2001). It has also been proposed that emission from jets (which are believed to be associated with 
the LH state) can account for the observed spectra in XRTs (e.g., Merloni \& Fabian 2002).

The XRT XTE J1118+480 was discovered by the All-Sky Monitor (ASM) on board of the Rossi X-ray Timing Explorer (RXTE) on 2000 March 29 (Remillard et al. 2000) as a weak, slowly rising X-ray source. Retrospective analysis of the ASM database revealed a previous outburst episode in 2000 January. The precursor was shorter than the main outburst, although both reached similar brightness (e.g., Uemura et al. 2000). XTE J1118+480 is one of the few XRTs that remained in a LH state throughout the outburst and failed to reach the HS state. This object is also important for several reasons. It is a secure case of a black hole $\left[f(M)=6.1 \pm 0.3 M_{\odot}\right.$; McClintock et al. 2001a; Wagner et al. 2001; Torres et al. 2004], the black hole transient with the shortest orbital period ( $P_{\text {orb }} \sim 4.1 \mathrm{hr}$; Patterson et al. 2000; Uemura et al. $2000)$ and also the first black hole binary in the Galactic halo (Wagner et al. 2001; Mirabel et al. 2001). In addition, the ratio $L_{\mathrm{X}} / L_{\mathrm{opt}} \simeq 5$ was extremely low, so it has been suggested that XTE J1118+480 may be an accretion disk corona source seen at high inclination (Garcia et al. 2000). However, no eclipse has ever been recorded, and consequently, the source should have been intrinsically faint in X-rays (Hynes et al. 2000) during the outburst. Finally, associated radio emission from this source has been reported (Pooley \& Waldram 2000) with jet interpretation (Fender et al. 2001).

The low interstellar absorption toward XTE J1118+480 allowed a detailed multiwavelength study during the 2000 outburst and quiescence, being the only XRT for which extreme ultraviolet observations could be achieved (Hynes et al. 2000; Chaty et al. 2003; McClintock et al. 2003). In fact, the best observations of an XRT in the LH state have been made for this system (together with GX 339-4). These observations have been of great significance in the effort to build a physical model of the accretion flow. XTE J1118+480 has been described in terms of an ADAF (McClintock et al. 2001b; Esin et al. 2001), an accretion disk corona model (Merloni et al. 2001), and a jet model (Markoff et al. 2001; Malzac et al. 2004; Yuan et al. 2005).

Here, we report the follow-up of a new outburst of the halo black hole X-ray transient XTE J1118+480 (hereafter J1118) and investigate the implications of modeling the structure of accretion flows onto black holes and the mechanisms involved.

\section{OBSERVATIONS AND REDUCTIONS}

Optical photometry was obtained with the $0.8 \mathrm{~m}$ IAC80 and the $1 \mathrm{~m}$ Optical Ground Station (OGS) telescopes at the Observatorio del Teide on Tenerife, the $1.52 \mathrm{~m}$ telescope at Loiano, the $1.2 \mathrm{~m}$ telescope at Fred Lawrence Whipple Observatory (FLWO), and the $4.2 \mathrm{~m}$ William Herschel Telescope (WHT) at the Observatorio del Roque de los Muchachos on La Palma. The target was mainly imaged in the $R$ band, although we also obtained some $B, V$, and $I$ colors. Integration times ranged from $10 \mathrm{~s}$ to 15 minutes, depending on telescope size, atmospheric conditions, and target brightness. All images were corrected for bias and flat-fielded in the standard way using IRAF. ${ }^{1}$ We performed aperture photometry on our object and several nearby comparison stars. We had previously performed a color-dependent calibration of a set of 14 stars in the $7^{\prime} \times 7^{\prime}$ field of view using several standard stars from six Landolt plates (Landolt 1992).

\footnotetext{
1 IRAF is distributed by the National Optical Astronomy Observatory, which are operated by the Association of Universities for Research in Astronomy, Inc., under cooperative agreement with the National Science Foundation.
}

Infrared photometry was obtained with the $1.3 \mathrm{~m} \mathrm{PAIRITEL}^{2}$ robotic telescope at FLWO. The camera is the Two Micron All Sky Survey (2MASS) South instrument, which images simultaneously in $J, H$, and $K_{s}$, covering a field of view of $8.5 \times 8.5$. A large number of dithered $7.8 \mathrm{~s}$ exposures are first bias and flatfield corrected and then combined to form mosaics for each individual observation, which is typically $600 \mathrm{~s}$ long (see also Blake et al. 2005). For each visit, instrumental $J H K_{s}$ magnitudes were extracted and consequently calibrated relative to the same five nearby 2MASS sources for all exposures. Photometric error estimates on the IR magnitudes are based on a combination of Poisson statistics and the error contribution of the five reference stars used for each observation.

The X-ray data were obtained from the RXTE ASM public archive, which contains several daily $2-12 \mathrm{keV}$ scans of the source since its first recorded X-ray outburst in 2000. Intensity measurements in three energy bands, $1.5-3,3-5$, and 5-12 keV, were also recorded. Before 2005 January 10, J1118 was never detected above the 10 mcrab 1 day bin sensitivity level (Levine et al. 1996). After January 16, the available data either do not yield good sensitivity measurements or they indicate that the flux is 10 mcrab or less.

\section{LONG-TERM LIGHT CURVE}

We discovered an optical rebrightening of J1118 $\sim 5 \mathrm{yr}$ after its first reported outburst (Zurita et al. 2005). We then embarked on a new campaign of systematic monitoring of the outburst light curve in the optical and infrared bands. The source reached $R=13.37 \pm 0.01$ at the outburst peak, $5.7 \pm 0.01$ mag above the mean quiescent level. In the infrared, we measured total outburst amplitudes of $4.9 \pm 0.1,5.0 \pm 0.1$, and $4.8 \pm 0.1$ mag in $J, H$, and $K_{s}$, respectively. Although the optical brightening is similar to that observed during the 2000 outburst, the current event did not power the source above $\sim 25$ mcrab in X-rays. Similarly, the 2000 outburst, although also faint in X-rays, reached a peak of $\sim 45$ mcrab (Wood et al. 2001). In Figure 1 we present our overall optical and infrared light curve of the 2005 outburst. The light curve morphology of both the X-ray and the optical emission is not the "canonical" fast rise and exponential decay (FRED; Chen, et al. 1997). In comparison, the precursor X-ray light curve for the 2000 outburst shows a FRED, and the March outburst (X-ray and optical) showed a plateau morphology (see the 2000 outburst light curves in, e.g., Wren et al. 2001). We note, however, that neither the sources that remained in a LH state nor a large number of those that reach the HS state are FRED shaped. We calculated the hardness ratio HR2 (5-12 keV : 3-5 keV) from the RXTE ASM data. Although only a few measurements were possible due to statistical limitations (see Fig. 1), we found $\mathrm{HR} 2=1.53 \pm 0.02$ at the peak of the outburst (from HJD $2,453,300=83$ to 86 ). This value of HR2 is consistent with a hard spectrum, supporting that J1118 likely remained in the LH state throughout the outburst (e.g., McClintock \& Remillard 2006).

The shape of the 2005 outburst light curve is remarkably similar to that of the "minioutbursts" observed in GRO J0422+ 32 (Chevalier \& Ilovaisky 1995) and XTE J1859+226 (Zurita et al. 2002b). About 30 days after the 2005 outburst peak, there is evidence for a short-lived reflare with amplitude of about 1 mag above the preevent level, lasting for about 10 days (see also the filled triangles in Fig. 1; Chou et al. 2005). Furthermore, the light

\footnotetext{
2 See http://www.pairitel.org.
} 


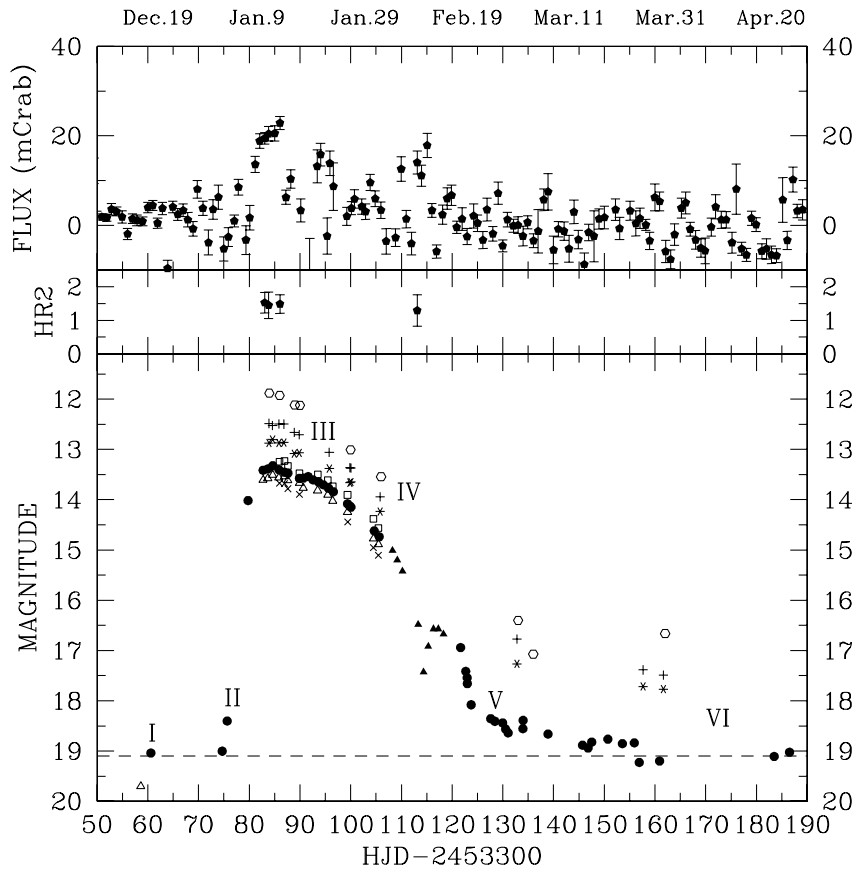

FIG. 1.-Long-term temporal evolution of J1118 plotted as (top to bottom): $\mathrm{X}$-ray flux (the X-ray data were provided by the RXTE ASM teams at MIT and at the RXTE SOF at NASA's GSFC); (5-12 keV):(3-5 keV) hardness ratio (HR2) derived from the RXTE ASM data; Optical magnitudes averaged per night, where optical and infrared data points are: $B$ (diagonal crosses), $V$ (triangles), $R$ (circles), $I$ (squares), $J$ (asterisks), $H$ (crosses), and $K_{s}$ (hexagons). Filled triangles mark the $V$-band magnitudes reported by Chou et al. (2005). The dashed line marks the mean quiescent magnitude in the $R$ band.

curve displays a small bump just before the system definitely settled down into quiescence. This type of behavior was also found during the minioutbursts of both GRO J0422+32 and XTE J1859+226 (see Fig. 2). These light curves have comparable length and brightness, and the X-ray-to-optical ratio measured in $\mathrm{J} 1118$ is also similar to what has been seen in minioutbursts, i.e., it is much lower than in normal outbursts (e.g., Zurita et al. 2002b). These facts support the suggestion of Hynes et al. (2000) that the outbursts in J1118 are indeed minioutbursts rather than full XRT events.

\section{OPTICAL MODULATION DURING EARLY DECLINE AND NEAR QUIESCENCE}

Example light curves of $\mathrm{J} 1118$ at different outburst epochs are presented in Figure 3. The data have been phase folded on the orbital ephemeris of Torres et al. (2004). Note the apparent changes in amplitude and morphology of the light curves as the outburst decays. We divide the light curve into six different stages (see also Fig. 1), preoutburst quiescence (I), rise (II), peak (III), decay (IV), near-quiescence (V), and postoutburst quiescence (VI).

I. On 2004 December 19 (UT) J1118 was still in quiescence and displayed the characteristic double-humped ellipsoidal modulation, with $\sim 0.16 \mathrm{mag}$ semiamplitude, driven by the tidally distorted secondary star.

II. On 2005 January 4, the outburst had already started and the $R$-band light curve shows the combination of the ellipsoidal modulation and a linear rise of $0.36 \mathrm{mag} \mathrm{day}^{-1}$.

III. The outburst peaks on January 14 and then the brightness in all bands started to decay at a moderately slow rate of $0.05 \mathrm{mag} \mathrm{day}^{-1}$. Near the outburst peak, on January 20 and 22,

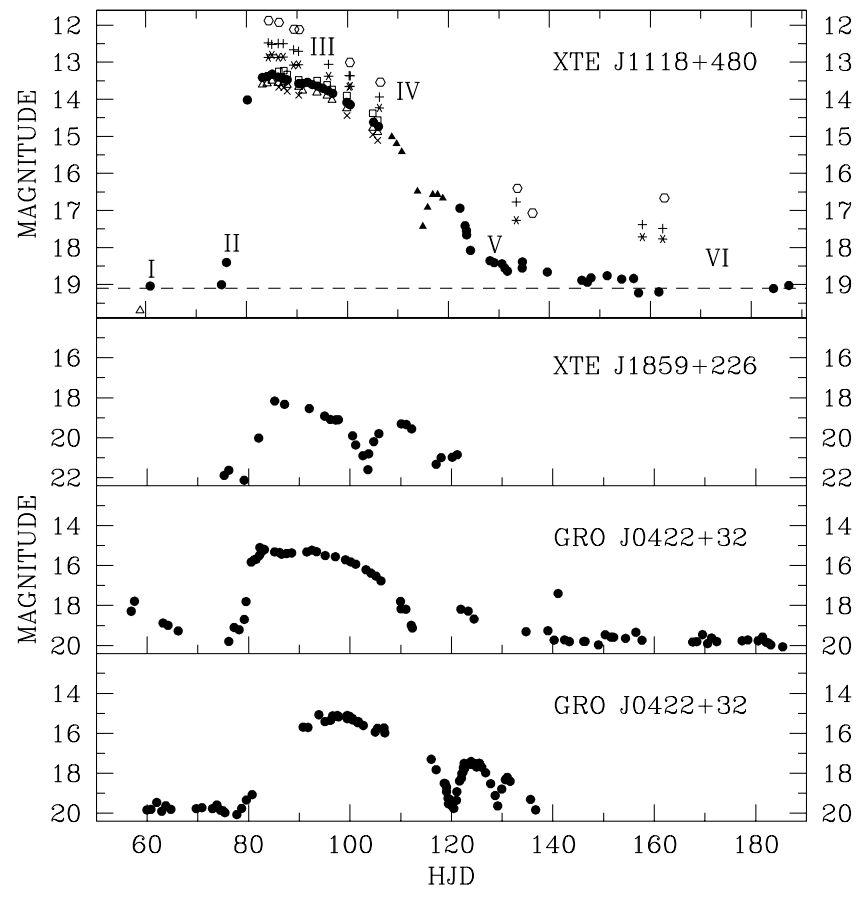

FIG. 2.-Top panel: Long-term optical light curve of J1118. Symbols for the optical and infrared data points are as in Fig. 1. Bottom panels: Minioutburst $R$-band light curves of XTE J1859+226 and GRO J0422+32 plotted for comparison (complete light curves of these systems can be found in Zurita et al. 2002b; Callanan et al. 1995). J1118 dates are HJD - 2,453,300, while XTE J1859+226 and GRO J0422+32 curves have been shifted in time to get the same minioutburst starting times. The J0422+32 data were provided by E. Kuulkers.



FIG. 3. $-R$-band light curves of J1118 taken during 2004 December-2005 April, covering the different outburst epochs, Preoutburst quiescence (I), rise (II), peak (III), decay (IV), near quiescence (V), and postoutburst quiescence (VI). They are phase folded on the ephemeris of Torres et al. (2004) and are shown twice for clarity. Note that the $y$-scale has been expanded when showing the January 20 and February 2 light curves. December 19, January 4, February 25, and April 26 light curves have been fitted with sinusoidal functions to simulate the ellipsoidal modulation. 



FIG. 4.-Top panels: Scargle periodogram of the $R$-band light curves obtained on January 20 and 22 (near the outburst peak, epoch III) after correcting for the overall decline and light curves folded on 0.156 days, corresponding to the peak marked on the Scargle periodogram. The data have been averaged into 200 phase bins. Bottom panels: Scargle periodogram of the $R$-band light curves obtained from March 17 to April 26 (postoutburst quiescence, epoch VI) and the light curves obtained on March 18 (open triangles) and April 26 ( filled circles), folded on 0.1699 days, corresponding to twice the peak marked on the Scargle periodogram. The solid line is the sinusoidal fit of all the epoch VI data, simulating the ellipsoidal modulation. Note the distortion of the two individual light curves with respect to the mean. A whole cycle is shown twice for continuity.

the $R$-band light curve shows a low-amplitude modulation superimposed on short-timescale $(\$ 5 \mathrm{~s})$ variability, likely due to flickering. This modulation was not observed after January 23 (alas, the $R$-band data obtained in January 21 was badly affected by weather conditions). The same modulation was also reported by Chou et al. (2005) in their $V$-band light curves obtained during January $18-20$. This indicates that the low-amplitude modulation was short-lived and/or its amplitude is diluted by the flickering or undetected due to the photometric accuracy. We searched for periodicities in the detrended light curves of January 20 and 22 by computing a Scargle periodogram. The result is shown in
Figure 4. The observing window produces an alias pattern at $\sim 6.5$ cycles day $^{-1}$, with the strongest peak at $0.156 \pm 0.002$ days. The 1 day alias centered at $0.169 \pm 0.003$ days lies close to both the orbital and superhump periods (see Uemura et al. 2000; Zurita et al. 2002a). Although the observed modulation is probably related to the orbital motion, the poor data sampling impedes an accurate period determination. In Figure 3 we show the January 20 light curve folded on the orbital period and averaged into 50 phase bins, although the cleanest modulation is seen when folding the data on 0.156 days (see Fig. 4, top left panel). 
IV. From the beginning of February the light curve began a moderately abrupt fall. We estimated a rate of $\sim 0.15$ mag day ${ }^{-1}$ in the $R$ band and a smoother slope of 0.10 mag day $^{-1}$ in the infrared bands. On February 2, no modulation is detected, only considerable flickering $\left(\sigma_{m} \sim 0.02 \mathrm{mag}\right)$, higher than that found at the peak of the outburst.

V. At the end of February, about 60 days after the outburst onset, J1118 settled into a near-quiescence level at $R=18.35 \pm$ 0.02 (Fig. 3). On February 25 the light curve is consistent with a (distorted) double-humped ellipsoidal modulation with a semiamplitude of $\sim 0.10 \mathrm{mag}$. We also note that the amplitude of the ellipsoidal modulation is lower at near-quiescence than at true quiescence before and after outburst. This is what we would expect if the contribution of the accretion disk to the total light is higher in February 25 (V) than in December 19 (I) and April 26 (VI). Assuming that the decrease in flux is solely due to the accretion disk light fading and that the disk contributed $\sim 55 \%$ to the total quiescent $R$-band light (Torres et al. 2004), we estimate a relative contribution of the accretion disk of $\sim 77 \%$ during epoch V.

VI. Two months later, J1118 faded another $0.6 \mathrm{mag}$ and reached $R \sim 19$ on March 17. The system remained at this level from there on, suggesting it reached true quiescence. At the same epoch we measured the following colors in the infrared: $K_{s}=$ $16.66 \pm 0.07, H-K_{s}=0.8 \pm 0.2$, and $J-K_{s}=1.1 \pm 0.1$. The $J$ and $K_{s}$ magnitudes are consistent with those observed by Mikołajewska et al. (2005) during the preoutburst quiescence (our epoch I). The colors $H-K_{s}$ and $J-K_{s}$ are much redder than expected for a later K or early M V secondary star, suggesting an additional contribution.

The Scargle periodogram of the epoch VI $R$-band light curves (March 17, 19, 20, and 23 and April 22 and 26) shows a strong peak centered on $0.0845 \pm 0.0005$ days (see Fig. 4 , bottom left panel), consistent with half the orbital period $P_{\text {orb }}=0.1699$ days. These light curves are also distorted, likely due to the presence of a superhump wave, as already noticed in the near-quiescence state at the end of the 2000 outburst (Zurita et al. 2002a). In contrast, on December 19 and January 4 (epochs I and II) there is no evidence of superhumps. This suggests that during outburst, the disk expands to the $3: 1$ (or $2: 1$ ) resonance radius and is then forced to precess by tidal perturbations caused by the secondary star. Afterward, it starts to shrink, although at early quiescence it is still large enough to produce superhump waves. Finally, some time later the disk radius becomes shorter than the resonance radius, and superhumps disappear.

\section{AN OPTICAL PRECURSOR TO THE X-RAY OUTBURST?}

There has been some evidence of optical/infrared outbursts starting before the X-ray outbursts in some XRTs, GS 1121-68 (Della Valle et al. 1991), GRO J1655-40 (Orosz et al. 1997), GRO J0422+32 (Castro-Tirado et al. 1997), V404 Cyg (Chen et al. 1997, and references therein), Aql X-1 (Shahbaz et al. 1998), J1118 during the main 2000 (March) outburst (Wren et al. 2001), and 4U 1543-47 (Buxton \& Bailyn 2004). In dwarf novae the UV rise has also been observed to start several hours after the optical outburst (e.g., Warner 1995, and references therein). In the framework of the "disk instability model" (see, e.g., Canizzo et al. 1995), the X-ray (or UV) delay suggests an "outside-in" disturbance of the accretion disk. Once the instability is triggered in the outer regions, a heating front propagates inward, turning the disk from the cold (quiescent) state to a hot state. Hence, the outburst is first noticed in the optical and then in X-rays (or
UV). The timescale of the lags can be explained assuming the accretion disk is truncated at some inner radius. The heating front stops when it arrives at the truncation radius, but the inner edge of the disk moves toward the compact object on the viscous timescale, longer than the front propagation time. The ADAF model can offer a natural explanation for the disk truncation in XRTs. Hence, it has been proposed that the disk inner truncation radius can be estimated by measuring the X-ray-to-optical delay (Hameury et al. 1997; Wren et al. 2001).

To take advantage of the fact that our optical observations covered part of the rise phase (see Fig. 1), we inspected whether the optical and X-ray outbursts were simultaneous or if one lags the other. The time-resolved optical light curve taken on 2005 January 4 (see Fig. 3) shows a linear rise. We therefore estimate the starting time for the optical rise to be $t_{\mathrm{opt}} \simeq 2,453,375.1 \pm 0.1$ (HJD) from a linear fit to this curve. A more problematic issue is to determine the starting time of the X-ray outburst. The X-ray light curve from ASM does not provide any useful information below the 10 mcrab sensitivity level, and hence extrapolation is required. We estimate that the X-ray outburst starting time is consistent with $t_{\mathrm{X}}=2,453,379.4 \pm 0.7$ (HJD), where the associated uncertainty quotes the differences between the several extrapolations we performed. This implies a $\sim 4$ day lag between the onset of the X-ray and optical outbursts. Unfortunately, the ASM sensitivity level is well above the quiescent flux level, making it very likely that any extrapolation overestimates the delay. Besides, most of the sources detected by the ASM need to brighten significantly above quiescence to be detected (see, e.g., Homan et al. 2005 for more details on this concern). Therefore, the true start of the X-ray outburst could be earlier than $t_{X}$. This fact forces us to conclude that the 4 day lag is just an upper limit, as it is the 10 day lag estimated by Wren et al. (2001). In short, although we can draw a qualitative picture of the evolution of the accretion disk in J1118 after the outburst, it is not possible to estimate a reliable preoutburst truncation radius from the X-ray delay using ASM data alone.

\section{THE SPECTRAL ENERGY DISTRIBUTION}

We constructed the spectral energy distributions (SEDs) for different outburst epochs through the outburst. The magnitudes were first corrected for interstellar extinction using $E(B-V)=$ 0.013 (Hynes et al. 2000) and the reddenings tabled in Rieke \& Lebofsky (1985), although this makes only a small difference in the dereddened magnitudes because the extinction is so low. Our outburst SEDs are shown in Figure 5. Here we have excluded some nights for clarity to avoid duplication. All SEDs are quasipower laws with $F_{\nu}$ increasing toward the blue. We have performed power-law fits to the optical SEDs only (from $\log \nu=$ 14.54 to 14.83 ), to the infrared magnitudes alone (from $\log \nu=$ 14.13 to 14.38 ), and then to the whole wavelength range (from $\log \nu=14.13$ to 14.83). The spectral indices we found have been plotted as a function of time and are shown in Figure 5 (bottom panel), where $F_{\nu} \propto \nu^{\alpha}$.

When considering the optical data alone (Fig. 5, open squares ), the source appears steeper (bluer) than the canonical $\alpha=1 / 3$ of a steady state viscously heated disk, being $\alpha=0.49 \pm 0.04$ at the peak of the outburst (from HJD $-2,453,300=85$ to 90 ). During the decay phase (IV), it becomes optically softer, with $\alpha \sim 0.25$. This trend is consistent with the cooling of the optically bright regions. However, when fitting the infrared alone, the trend is reversed, showing that the infrared evolves very differently (open triangles) than the optical. The joint fit (filled circles) is the interplay between these components and shows an exponential trend becoming bluer throughout the outburst. At the 



FIG. 5.-Top panel: Evolution of the optical-infrared SEDs for J1118 through the outburst. Different symbols are used to distinguish alternate epochs and solid lines are power-law fits. Numbers label HJD*=HJD-2,453,300 (note that the optical outburst started at $\mathrm{HJD}^{*}=75$ ). Bottom panel: Evolution of the powerlaw index, $\alpha$ (where $F_{\nu} \propto \nu^{\alpha}$ ). Open squares are the indices obtained from the optical SEDs (from $\log \nu=14.54$ to 14.83), open triangles correspond to the infrared SED fits (from $\log \nu=14.13$ to $\log \nu=14.38$ ), whereas filled circles are the indices obtained from the optical-infrared SED fits (from $\log \nu=14.13$ to 14.83). The dotted line corresponds to the canonical $\nu^{1 / 3}$ value for a steady state viscously heated disk. The dashed line shows the exponential fitting to the indices obtained from the whole wavelength range SEDs.

decay phase the index is consistent with $\alpha=1 / 3$. We discuss the implications of the different SEDs in the next section.

\section{SUMMARY AND DISCUSSION}

We present the follow-up of the new outburst of the halo black hole X-ray transient XTE J1118+480. We estimate a total outburst amplitude of $5.7 \pm 0.1$ in the $R$ band and $\sim 5$ mag in the $J$, $H$, and $K_{s}$ bands. The shape of the light curve is remarkably similar to the minioutbursts observed in GRO J0422+32 and XTE J1859+226. Furthermore, $L_{\mathrm{X}}(1-10 \mathrm{keV}) / L_{\mathrm{opt}} \sim 5$, similar to minioutburst episodes, whereas typically this ratio is $\sim 500$ in normal X-ray transient outbursts.

Quiescent XRTs are often explained by assuming an accretion disk extending down to a certain transition radius, while the inner volume is filled with a hot ADAF (e.g., Narayan et al. 1996). This model predicts that after outburst, the inner disk edge will move further inward. Because the efficiency of energy release in the ADAF region is very low, the X-ray outburst starts when the densest parts of the disk can penetrate far enough in to allow an efficient transformation of gravitational energy into radiation. The outer disk is also expected to change. In the course of the outburst, matter diffuses inward, closing the disk, while angular momentum transferred to the outer parts forces the outer radius to increase. If it reaches the 3:1 resonance radius, the disk will precess by tidal interaction with the secondary star (e.g., Whitehurst \& King 1991), and a superhump modulation will presumably be visible. This was the case in 2000 March when the change in the outer disk radius could be measured from the change in the superhump period at different stages of the outburst (Zurita et al. 2002a). Likewise, in 2005 we found a 0.02 mag amplitude variation during the early decline and a distorted double-humped ellipsoidal modulation during the near-quiescence level and true quiescence. This suggests that the disk expanded after outburst to the 3:1 resonance disk radius, where it remained during the early phases of quiescence.

Recently, the LH state of X-ray binaries has been associated with jet activity. In some cases a jetlike structure has been resolved (e.g., Mirabel et al. 1992). When jets cannot be directly imaged, a flat or even inverted radio spectrum is often considered to be a typical signature of jet emission (Fender et al. 2001). However, it is also apparent that the jet contributes outside the radio band. The synchrotron spectrum, which is thought to be the jet signature, is frequently seen at radio but also up to higher frequencies in the infrared and possibly in the optical. In the case of J1118, the SED from radio to X-rays during the 2000 outburst has been explained as a combination of synchrotron radiation from a jet and a truncated optically thick disk (Hynes et al. 2000; Markoff et al. 2001; Yuan et al. 2005), whereas models assuming ADAF alone (McClintock et al. 2001b; Esin et al. 2001) underestimated the optical and the infrared fluxes. The spectrum from infrared to UV is flat ( $F_{\nu} \sim$ const; Hynes et al. 2000), although the optical spectrum alone has blue continuum slopes of $\alpha=1 / 3$, as expected for an optically thick accretion disk (Dubus et al. 2001; Torres et al. 2002).

The SEDs during the 2005 outburst exhibit quasi-power-law spectra with $\alpha$ softening from $\sim 0.49$ during the peak of the X-ray outburst to $\sim 0.25$ during the decay phase. However, when fitting the infrared alone, we find a flat spectrum with $\alpha=0.1 \pm$ 0.1 at the outburst peak. This difference between the optical and the infrared SEDs, more important at the outburst peak, suggests that the infrared is dominated by a different component (a jet?), whereas in the optical, we are presumably seeing the disk evolution. The very flat infrared SED $\left(F_{\nu} \sim\right.$ const) could naturally be interpreted as a mixture of an optically thick disk spectrum and flat-spectrum emission, possibly synchrotron. Linear fits to optical SEDs have also been performed for other short-period black hole XRTs in outburst (see the compilation by Hynes 2005). The optical SEDs for the Hynes (2005) relatively uniform set, exhibit quasi-power-law spectra with $\alpha$ ranging between 0.5 and 1.5 , all steeper than the canonical $F_{\nu} \propto \nu^{1 / 3}$. Two of the sources among the Hynes (2005) sample (GRO J0422+32 and XTE $\mathrm{J} 1859+226)$ were identified by Brocksopp et al. (2004) as hard sources. (XTE J1859+226 is hard, at least early in the outburst.) The spectra of both sources exhibit a quasi-exponential softening throughout the outburst, whereas the other systems exhibit no clear trends. However, very little data are available for these two systems.

Our data clearly demonstrate the added value of extending the wavelength range into the near-infrared. We were able to witness additional spectral components that show a different trend during the course of the outburst. Extending the wavelength coverage even further would have allowed for a more quantitative comparison with proposed descriptions of the accretion flows near compact objects. Looking ahead, simultaneous multiwavelength observations from X-rays through to radio will enable us to validate the interplay between disks and jets. J1118 remains an excellent target for multiwavelength studies that needs to be exploited with future and present facilities as we look forward to its next outburst.

M. A. P. T. thanks the observers at the $1.5 \mathrm{~m}$ telescope at FLWO (in particular Perry Berlind and Mike Calkins) for helping during the remote observations with the $1.2 \mathrm{~m}$. This work was supported 
by NASA LTSA grant NAG-5-10889. D. S. acknowledges a Smithsonian Astrophysical Observatory Clay Fellowship. The Peters Automated Infrared Imaging Telescope (PAIRITEL) is operated by the Smithsonian Astrophysical Observatory (SAO) and was made possible by a grant from the Harvard University Milton Fund, the camera loan from the University of Virginia, and the continued support of the SAO and the University of California, Berkeley.
Blake, C. E., et al. 2005, Nature, 435, 181

Brocksopp, C., Bandyopadhyay, R. M., \& Fender, R. P. 2004, NewA, 9, 249

Buxton, M. M., \& Bailyn, C. D. 2004, ApJ, 615, 880

Callanan, P. J., et al. 1995, ApJ, 441, 786

Cannizzo, J. K., Chen, W., \& Livio, M. 1995, ApJ, 454, 880

Castro-Tirado, A. J., Ortiz, J. L., \& Gallego, J. 1997, A\&A, 322, 507

Charles, P. A., \& Coe, M. J. 2006, in Compact Stellar X-Ray Sources, ed. W. H. G. Lewin \& M. van der Klis (Cambridge: Cambridge Univ. Press), in press (astro-ph/0308020)

Chaty, S., Haswell, C. A., Malzac, J., Hynes, R. I., Shrader, C. R., \& Cui, W. 2003, MNRAS, 346, 689

Chen, W., Shrader, C. R., \& Livio, M. 1997, ApJ, 491, 312

Chevalier, C., \& Ilovaisky, S. A. 1995, A\&A, 297, 103

Chou, Y., Chen, A., Chiang, P. S., Hu, C. P., \& Hu, R. H. 2005, Astron. Tel., 412,1

Della Valle, M., Jarvis, B. J., \& West, R. M. 1991, A\&A, 247, L33

Dubus, G., Kim, R. S. J., Menou, K., Szkody, P., \& Bowen, D. V. 2001, ApJ, 553, 307

Esin, A. A., McClintock, J. E., Drake, J. J., Garcia, M. R., Haswell, C. A., Hynes, R. I., \& Muno, M. P. 2001, ApJ, 555, 483

Fender, R. P., Hjellming, R. M., Tilanus, R. P. J., Pooley, G. G., Deane, J. R., Ogley, R. N., \& Spencer, R. E. 2001, MNRAS, 322, L23

Garcia, M., Brown, W., Pahre, M., McClintock, J., Callanan, P., \& Garnavich, P. 2000, IAU Circ., 7392, 2

Hameury, J. M., Lasota, J. P., McClintock, J. E., \& Narayan, R. 1997, ApJ, 489, 234

Homan, J., Buxton, M., Markoff, S., Bailyn, C. D., Nespoli, E., \& Belloni, T. 2005, ApJ, 624, 295

Hynes, R. I. 2005, ApJ, 623, 1026

Hynes, R. I., Mauche, C. W., Haswell, C. A., Shrader, C. R., Cui, W., \& Chaty, S. 2000, ApJ, 539, L37

Landolt, A. U. 1992, AJ, 104, 340

Levine, A. M., Bradt, H., Cui, W., Jernigan, J. G., Morgan, E. H., Remillard, R., Shirey, R. E., \& Smith, D. A. 1996, ApJ, 469, 33L

Malzac, J., Merloni, A., \& Fabian, A. C. 2004, MNRAS, 351, 253

Markoff, S., Falcke, H., \& Fender, R. 2001, A\&A, 372, L25

McClintock, J. E., Garcia, M. R., Caldwell, N., Falco, E. E., Garnavich, P. M., \& Zhao, P. 2001a, ApJ, 551, L147

McClintock, J. E., Narayan, R. Garcia, M. R., Orosz, J. A., Remillard, R. A., \& Murray, S. S. 2003, ApJ, 593, 435

\section{EFERENCES}

McClintock, J. E., \& Remillard R. A. 2006, Compact Stellar X-ray Sources, ed. W. H. G. Lewin, \& M. van der Klis (Cambridge: Cambridge Univ. Press), in press (astro-ph/0306213)

McClintock, J. E., et al. 2001b, ApJ, 555, 477

Merloni, A., Di Matteo, T., \& Fabian, A. C. 2001, Ap\&SS Suppl., 276, 213

Merloni, A., \& Fabian, A. C. 2001, MNRAS, 321, 549 2002, MNRAS, 332, 165

Mikołajewska, J., Rutkowski, A., Gonçalves, D. R., \& Szostek, A. 2005, MNRAS, 362, L13

Mirabel, I. F., Dhawan, V., Mignani, R. P., Rodrigues, I., \& Guglielmetti, F. 2001, Nature, 413, 139

Mirabel, I. F., Rodriguez, L. F., Cordier, B., Paul, J., \& Lebrun, F. 1992, Nature, 358,215

Narayan, R., McClintock, \& J. E., \& Yi, I. 1996, ApJ, 457, 821

Orosz, J. A., Remillard, R. A., Bailyn, C. D., \& McClintock, J. E. 1997, ApJ, 478, L83

Patterson, J., et al. 2000, IAU Circ., 7412, 2

Pooley, G. G., \& Waldram, E. M. 2000, IAU Circ., 7390, 2

Remillard, R., Morgan, E., Smith, D., \& Smith, E. 2000, IAU Circ., 7389, 2

Rieke, G. H., \& Lebofsky, M. J. 1985, ApJ, 288, 618

Shahbaz, T., Bandyopadhyay, R. M., Charles, P. A., Wagner, R. M., Mulhi, P., Hakala, P., Casares, J., \& Greenhill, J. 1998, MNRAS, 300, 1035

Torres, M. A. P., Callanan, P. J., Garcia, M. R., Zhao, P., Laycock, S., \& Kong, A. K. H. 2004, ApJ, 612, 1026

Torres, M. A. P., et al. 2002, ApJ, 569, 423

Uemura, M., et al. 2000, PASJ, 52, L15

Wagner, R. M., Foltz, C. B., Shahbaz, T., Casares, J., Charles, P. A., Starrfield, S. G., \& Hewett, P. 2001, ApJ, 556, 42

Warner, B. 1995, Cataclysmic Variable Stars (Cambridge: Cambridge Univ. Press)

Whitehurst, R., \& King, A. 1991, MNRAS, 249, 25

Wood, K. S., Titarchuk, L., Ray, P. S., Wolff, M. T., Lovellette, M. N., \& Bandyopadhyay, R. M. 2001, ApJ, 563, 246

Wren, J., et al. 2001, ApJ, 557, L97

Yuan, F., Cui, W., \& Narayan, R. 2005, ApJ, 620, 905

Zurita, C., et al. 2002a, MNRAS, 333, 791 2002b, MNRAS, 334, 999

2005, Astron. Tel., 383, 1 